\title{
ECG Signal De-noising based on Adaptive Filters
}

\author{
Keshavamurthy T G, M. N. Eshwarappa
}

\begin{abstract}
Denoising a signal is one of the most important tasks in signal processing. Electrocardiogram (ECG) test gives more efficient result to analyze the heart diseases. The amplitude and frequency of the ECG signals are added with various noises and that may lead to a wrong analysis of ECG or it is difficult to interpret and quality is degraded. In this paper three different noises are added to raw ECG signal, Power-line Interference noise (PLI), Baseline Wandering (BW) noise and Composite Noise (CN). The noisy signal is pre-processed using bandpass filter, low-frequency ECG signal is selected by applying DWT, CEEMD (Complementary Ensemble Empirical Mode Decomposition), LMS (Least Mean Square) and NLMS (Normalized Least Mean Square) are the different filtering techniques used to denoised. To increase the signal quality, the denoised ECG is applied to Kalman Smoother. Inverse wavelet transforms, which reconstruct signal without destructing features of ECG signal. The simulation result shows that the proposed system with better performance compared to another traditional system in terms of Signal to Noise Ratio (SNR), Correlation Coefficient (CCR), Percentage Root mean square Difference (PRD) and the Mean Square Error (MSE).
\end{abstract}

Keywords: ECG, CEEMD, LMS, NLMS, DWT, IDWT.

\section{INTRODUCTION}

ECG is a widely utilized tool for the identification of cardiovascular disorders. A typical ECG contains the following segments, namely P, Q, R, S, and T wave. Due to the increased growth of population, computer-based automated ECG analyzer has great importance. For reliable and efficient analyzing of ECG, a noise-free ECG signal is much desired [18]. However, practically during acquisition and transmission, several noises, in particular, WGN, PLI, muscle artifact, baseline wander, etc. contaminates with ECG signal. White Gaussian Noise (WGN) is generated because of the poor channel condition, PLI appears because of power supply, muscle artifact is introduced by the muscle activity and baseline wander is occurred due to respiration [15]. Removal of these noises is essential for analysis. Hence, the denoising of ECG signal using efficient algorithms is an important [19].

Waveforms of ECG are generated by placing the electrode on the body, which contains all information about the heart health condition [11]. Amplitude various due to the variation in the potential difference from unexcited muscles to excited muscles due to the depolarization of heart muscle and polarization of heart muscle which can be analyzed and displayed. ECG waveform consists of $\mathrm{P}, \mathrm{Q}, \mathrm{R}, \mathrm{S}, \mathrm{T}$ wave and sometimes $\mathrm{U}$ wave as shown in figure 1 .

Revised Manuscript Received on November 08, 2019.

Keshavamurthy T G, Assistant Professor, Department of TCE Sri Siddhartha Institute of Technology, Tumkur, Karnataka, India

Dr. M. N. Eshwarappa, Professor, Department of ECE Sri Siddhartha Institute of Technology, Tumkur, Karnataka, India

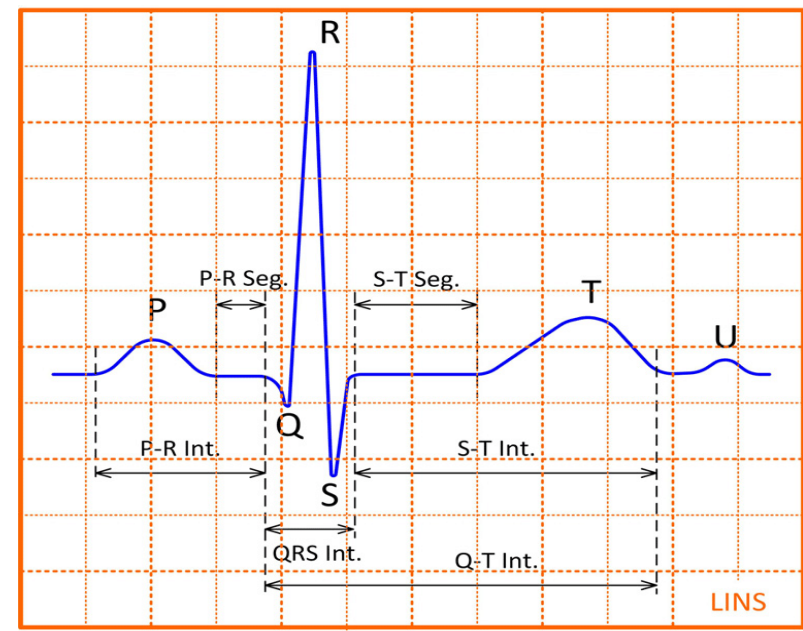

Figure 1: ECG Signal Waveform

This waveform contains different amplitudes and time duration $\mathrm{P}$ and $\mathrm{T}$ waves are low-frequency short signals $\mathrm{R}, \mathrm{S}$ and Q signals forming QRS complex. Time is measured by the width of the wave. Voltage is measured by the height and depth of the signal. All parameter are determined to determine the cardiac status of the patient's, QRS and U wave are taken for biometric analysis. If any noise is present in that waves it changes its time duration and amplitude of the QRS segment as shown in Figure 2.

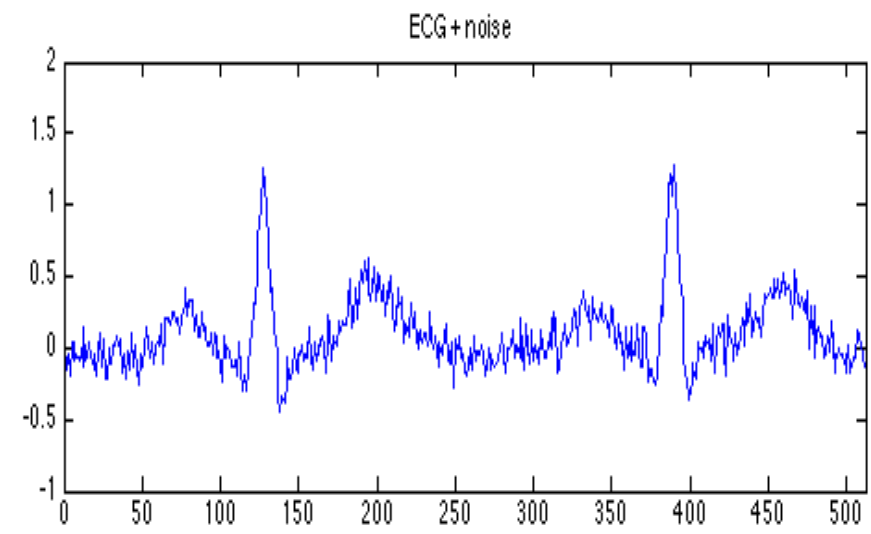

Figure 2: ECG with noise

ECG taken by placing ECG leads on the body of the patient, the signal is affected by various noise by action of the patient, drift in the baseline, interference of the power line, etc. if the signal is affected by the noise, signal is not visible properly, denoising the signal to use it in the further analysis is one of the major tasks. ECG includes different types of noise such as electrode-skin contact and electrical movement of muscles creates muscle noise. Cables connected to power line creates $\mathrm{AC}$ current due to the loops in the cable it creates power line noise. Combining different type's noise creates $\mathrm{CN}$. The noises can be filtered by using effective signal denoising method which increases the performance. The signal performance can be analyzed by SNR, MSE, CCR and PDR parameters. 


\section{ECG Signal De-noising based on Adaptive Filters}

In this paper, there are various types of noise are added to ECG, noise synthetic signals are generated using equation. The original raw ECG is added with the generated synthetic noise signal, in the preprocessing to select only the desired band of signal, a bandpass filter is applied. DWT is applied and low-frequency signal band is selected, the output of DWT is denoised using different denoising techniques like CEEMD, LMS, and NLMS. Kalman smoothening is applied to the denoised signal. Inverse wavelet transformation is applied to reconstruct the signal.

The paper organization is as follows: section II explains Literature Survey, section III explains Methodology, section IV explains Experimental Results and section V explains Conclusion.

\section{LITERATURE SURVEY}

Fakheraldin Y. O et.al [01] has presented a novel strategy on CEEMDAN to denoise the ECG influenced by PLI. The performance of the system is analyzed. The ECG always nonstationary signal and it is nonlinear, hence the signal is decomposed using CEEMDAN to IMFs, the first IMFs of the signal is considered as a reference to different adaptive filters. The performance parameters show that the system gives good result compare with the recently developed system.

Xiaoran Ning et.al [02] proposed noise elimination from the ECG signal. Enhancing ECG using Sparse Derivative Denoising (SDD), ECG is divided into three components where sparse components are present in the first two, the third component includes only noise. With the help of Variable splitting and ADMM, the convex optimization problem is solved.

Uzzal Biswas et.al [03] has proposed a system to remove the noise in ECG which is affected by a different type of noise. Two adaptive filters are used to denoise the signal, LMS and NLMS. The results of the system are compared, PSD, SNR, Mean Square Error (MSE) are the parameters considered to evaluate the performance and it proves that the adaptive NLMS filters give good performance in denoising different noise.

Shuicai Wu et.al [04] Proposed ECG signal for feature extraction. they took two signals for input one is the original signal which is abdominal signal and another one is a reference signal which is a thoracic signal is filtered by using LMS and SSNF algorithm which removes the noise.

Shivani Sharma et.al [05] has presented a system which denoise the signals. ECG is a significant analysis test to know about the condition of the human heart. ECG is easily be corrupted by noise. LMS and NLMS filters are applied to denoise the ECG. MATLAB simulation is used to compare the performance of the proposed system. The ECG samples are considered from the MIT-BIH standard database and white WGN is added to the input original ECG. SNR calculated as a performance parameter to analyze and shows the improved SNR value.

Motahar Hossain Mishu et.al [06] has presented denoising of ECG. They used the dual-tree complex wavelet transform (DT-CWT) for removing the noise. The result shows that SNR is high for a de-noised signal when compared to the original signal.

Sarang L. Joshi et.al [07] proposed a different de-noising technique for ECG signal. The best method to remove BW using empirical mode decomposition. The best method to remove PLI noise by Equiv ripple notch filter and to remove EMG and motion artifact by discrete Meyer wavelet transform.

Deboleena Sadhukhan et.al [08] proposed noise removal of ECG signal. Low and high-frequency noise are eliminated using a linear filter and non-linear filter. Lowfrequency noise is present in linear filter present which is having $\mathrm{BW}$ as a noise parameter. High-frequency noise is present in a non-linear filter which is having different noise parameter. Their method gives high SNR ratio compared various types of noise.

Jenitta et.al [09] has proposed a system based on the adaptive filter with EMD and Ensemble Empirical Mode Decomposition (EEMD) to denoise the ECG signal. The signal is decomposed into a number of IMFs using EEMD, the decomposed signal is denoised using Block Least Mean Square Error (BLMS). The performance parameter signal to noise ratio, correlation coefficient and mean square error is analyzed to and compare with the recently developed systems. EEMD with BLMS gives better than the traditional EMD based methods.

\section{METHODOLOGY}

ECG machine noninvasively records the electrical activity of the heart. The electrodes of the ECG device placed on the chest to record. The ECG is a significant test in denoising the cardiac diseases. But while recording the ECG there are chances that the signal is corrupted by different noise like PLI, BW, and CN. Due to noise in the ECG, the accuracy of the signal is degraded. It is required to attenuate the noise in the ECG for more perfect diagnosis of a heart condition.

Figure 3 shows the proposed system architecture to eliminate noise in the ECG signal. The raw signal obtained by the clinical database is added with the PLI, BW noise and $\mathrm{CN}$. The noise signal is created using an equation, those signals are called synthetic signals. At the pre-processing stage, only a selected band of frequency signal is selected using a bandpass filter. The signal is decomposed using DWT into a different band of frequency, from DWT the low-frequency signal is selected for further processes. Different adaptive filters such as CEEMD, LMS, and NLMS are used to remove all types of noise. Composition of the signal by using Inverse Discrete Wavelet Transforms (IDWT) which reconstruct signal without affecting any features of the signal. Each block is briefly explained below. 


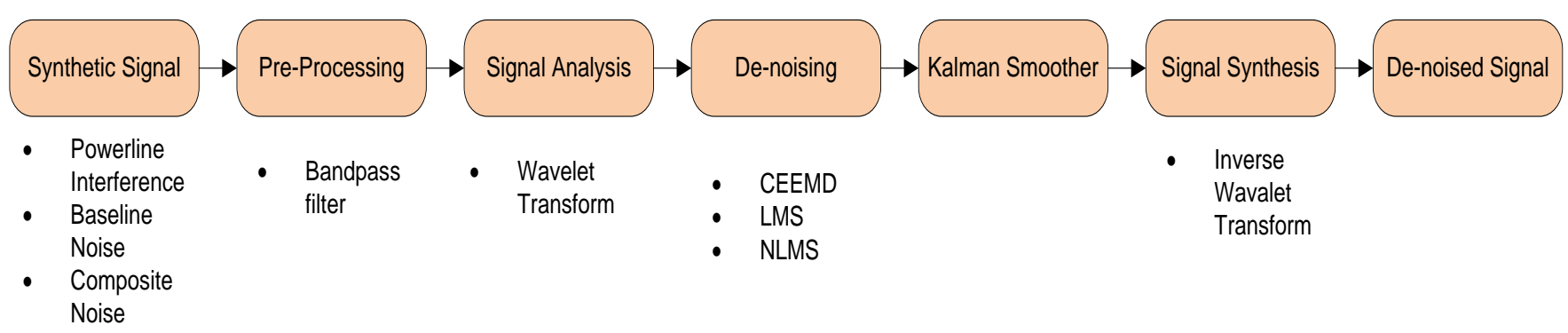

Figure 3: Proposed System Block Diagram

\subsection{Synthetic signal}

The synthetic signal is an input signal which consists of different type's noise such as PLI, BW noise, and CN.

A. $P L I$

$50 \mathrm{~Hz}$ to $60 \mathrm{~Hz}$ sinusoidal signal generates the power line noise due to AC PLI [14]. Its amplitude is exactly half the percentage of peak-to-peak ECG amplitude [10].

Some reasons for PLI

- Cables connected to PL creates alternating current due to the loops in the cable

- ECG machine ground connection

- Electrode disconnection

B. $B W$

It is a supplementary noise in the ECG where the base of any ECG appears to "wander" or move up and down instead of being straight [13]. It is usually caused due to body actions, improper electrodes, sweat, respiration or movement of the patient. Though its spectral content is below $1 \mathrm{~Hz}$, sometimes amplitudes of wander exceed the amplitude of QRS component nearly in between the range of $0.15-0.3 \mathrm{~Hz}$ [23].

Among these noises, the PLI and the BW noise are the most serious one and can strongly affect the ECG accordingly.

\subsection{Pre-processing}

The signals are considered from the standard database. In the pre-processing, the selected ECG is filtered. During ECG acquisition signal may affect by noise. The input ECG signal ranges are selected between 1 to 1000 samples and bandpass filter is applied to eliminate the unwanted signal and to select only particular band of signal for the next process.

\subsection{Signal Analysis}

Applying the wavelet transforms to the pre-processed signal gives the resolution in both time and frequency. The wavelet coefficients performance characteristics of the signal in each scale are different from noise. It can discard the noise wavelet components and retain signal wavelet coefficients. Then it can reconstruct the original signal.

\subsubsection{Wavelet Selection}

Wavelet function is not the only one, all the function which satisfies the conditions can be as a wavelet function [12]. The general principles of wavelet selection are mainly in the following

- Wavelet support set length, reflecting the speed of wavelet convergence when the time or frequency tended to infinity

- Wavelet vanishing moments, it is very useful for data compression, the actual impact of vanishing moments is to make the signal energy concentrated in a relatively small number of wavelet coefficients, reflecting the characteristics of the wavelet energy concentration
- Symmetry properties, related to the linear phase of wavelet filter, with relation to distortion issues

- Regularity, which is very significant for signal reconstruction and obtaining better smoothing effect, embodies the localization study of the wavelet transform.

In the processing of the ECG, the requirements for realtime and phase shift are not very important. So the considerations to support set length and symmetry can be ignored. The most important is the wavelet regularity.

Representing the ECG in both time and frequency domain. Location $b$ and scaling $a$ are defined to get a function and is given by Eq. (1)

$$
\varphi_{(a, b)}(t)=\frac{1}{\sqrt{a}} \varphi\left(\frac{t-b}{a}\right)
$$

For obtaining the DWT the sampling of wavelet transform is done on the dyadic grid scale. For every time instant, up to $n$ DWT is applied at scale $2^{-m}$ and is given by Eq. (2)

$$
\varphi_{(m, n)}(t)=2^{-\frac{m}{2}} \varphi\left(2^{-m} t-n\right)
$$

Representing DWT in the form of the inner product between the signal by using dyadic wavelets function and is given by Eq. (3)

$$
T_{(m, n)}=\int_{-\infty}^{\infty} x(t) \varphi_{(m, n)}(t) d t
$$

\subsection{Denoising}

Removing the noise is performed by different adaptive filters such as CEEMD, LMS, and NLMS, are explained below.

A. CEEMD

Applying CEEMD algorithm to the ECG signal. CEEMD divide the given signal into intrinsic modes functions (IMF) and them oscillatory components which include higher frequencies and lower frequency. For making uniform scaling add white noise to original signal $\mathrm{x}$ then performing EMD. Each white noise is sampled twice for CEEMD. N number of WGN can be collected and is given by Eq. (4)

$$
\leq N \quad\left(n_{i}\right) \text { for } 1 \leq i
$$

WGN is added to both positive and negative amplitude of original ECG and they are $x+n_{i}$ and $x-n_{i}$. for N WGN $\mathrm{N}$ IMFs are calculated then the average IMF gives the true IMF. 


\section{ECG Signal De-noising based on Adaptive Filters}

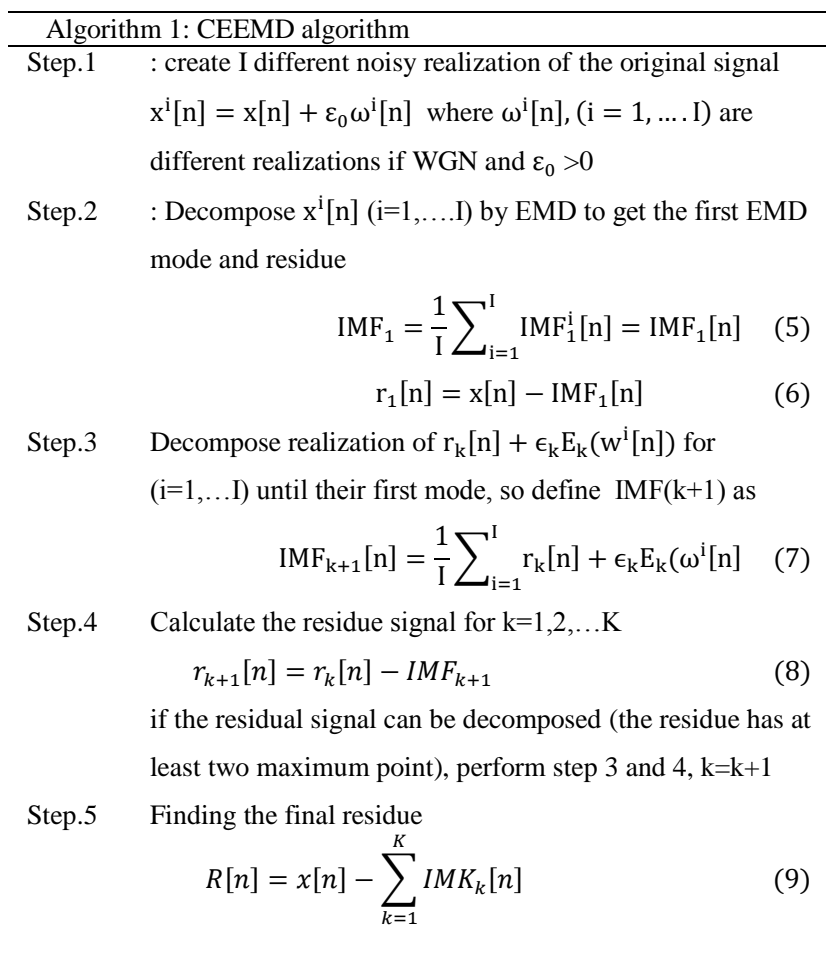

B. $L M S$

LMS algorithm removes noise present in the signal by taking filter coefficients. It removes low-frequency noise in signal [11] [17]. Convergence behavior is the important property of LMS algorithm in a stationary environment. LMS is a linear adaptive filtering algorithm. Figure 4 is a flow chart of the LMS algorithm.

Weight Updating for LMS algorithm is given by Eq. (10)

$$
w(n+1)=w(n)+2 \mu e(n) x(n)
$$

Where the adaptive filter coefficient is $w(n)$ and $\mu$ is step size. The abdominal signal $d(n)$ as the raw input at the nth moment is given by Eq. (11)

$$
d(n)=[d(n), d(n-1), \ldots d(n-m+1)]^{T}
$$

Where $m$ is the length of the adaptive filter and the thoracic signal $x(n)$ as there reference input at the nth moment is given by Eq. (12)

$$
\begin{aligned}
& x(n)=[x(n), x(n-1), \ldots . x(n-m \\
+1)]^{T} & (12)
\end{aligned}
$$

The filtered output signal $y(n)$ which is close to the maternal ECG will be given by Eq. (13)

$$
y(n)=\sum_{i=0}^{m-1} w_{i} x(n-i)=w^{T}(n) x(n)
$$

Then Person ECG can be obtained after adaptive processing and is given Eq. (14)

$$
e(n)=d(n)-e(n)=d(n)-w^{T}(n) x(n)
$$

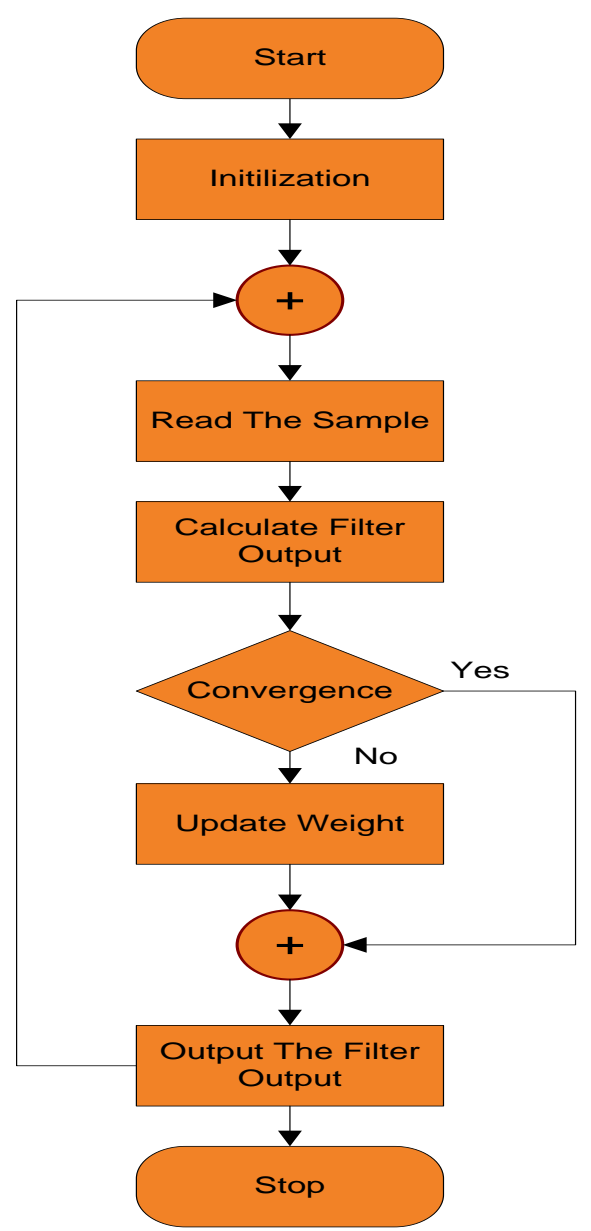

Figure 4: LMS Flow Chart

C. $\quad N L M S$

Adaptive coefficients are trained by the NLMS algorithm [19]. This algorithm is used for varying step size signal by making it to normalized step size which gives the stable signal.

Updated weights for NLMS filter is given below Eq. (15)

$w(n+1)=w(n)+\left[\frac{\mu}{p+x^{t}(n) x(n)}\right] x(n) \cdot e(n)(15)$

The variable step is given by Eq. (16)

$$
\mu(n)=\left[\frac{\mu}{p+x^{t}(n) x(n)}\right]
$$

NLMS is higher version LMS where normalized weights are taken. NLMS converge faster compared to LMS which minimizes the error rate.

\subsection{Kalman smoother}

After applying the Kalman filter, Kalman smoother is applied for a linear system. If it is nonlinear system Extended Kalman Filter (EKF) is applied then Extended Kalman Smoother (EKS) is applied.

EKS is applied after EKF. It includes a forward EKF stage followed by a backward recursive smoothing stage. Since EKS includes information by considering futures aspects. It gives better results compared to the ECG signal which gives more accurately than EKF in noisy scenarios. Finally, EKS makes smoother results if signals lagging also.

\subsection{Signal Synthesis}

Finally, denoised signals are reconstructed without destructing features of the signal. The reconstruction is done by performing the IDWT of various wavelet coefficients for each decomposition level. 
IDWT is applied for Eq. (2) than Eq. (17) is obtained and is given below.

$$
x(t)=\sum_{m=-\infty}^{\infty} \cdot \sum_{n=-\infty}^{\infty} T_{(m, n)} \varphi_{(m, n)}(t)
$$

Finally, the denoised signal is obtained by applying different types of filtering method.

\section{EXPERIMENTAL RESULTS}

The system evaluated using MATLAB R2010a tool. The raw ECG signal dataset is considered from the standard MIT-BIH dataset. Generated noise synthetic signal added with ECG is considered as an input. the noisy ECG is pre-processed using bandpass filtered and decomposition of the signal by wavelet transform Then de-noising the filtered signal is by using CEEMD, LMS and NLMS de-noising technique. The noise removed signal is applied for Kalman smoother which gives a smooth signal. Reconstruct the denoised signal by IDWT. Finally, the noise removed signal is obtained.

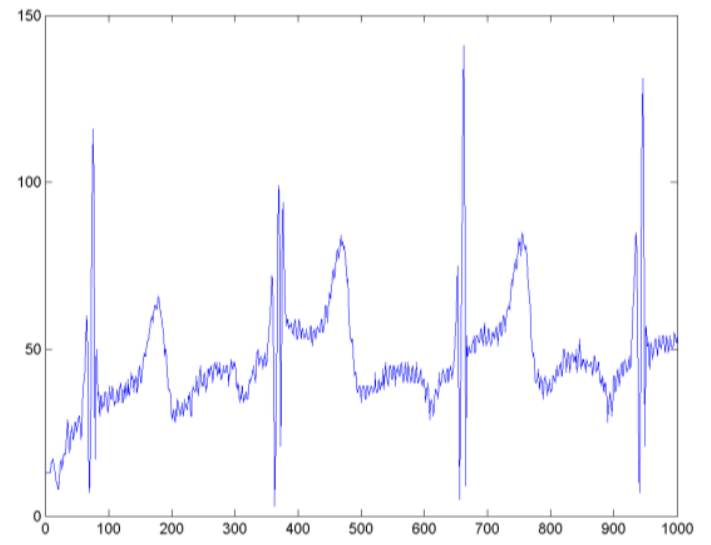

(a) Input Signal

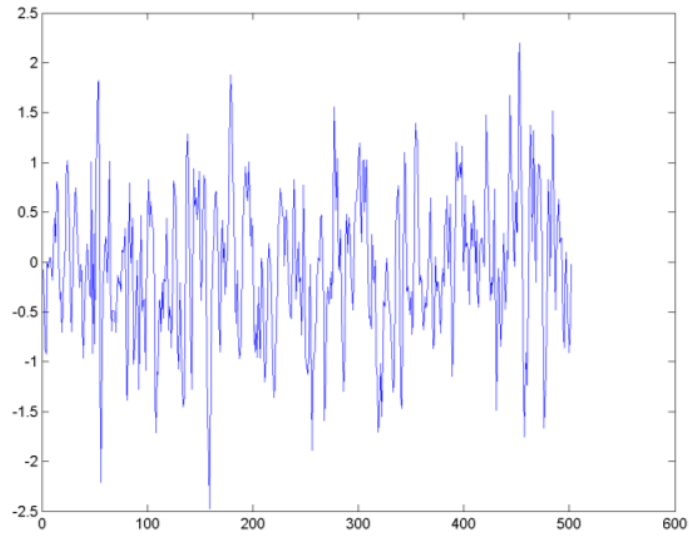

(c) NLMS output signal

Figure 5 shows the results of PLI noise denoising using NLMS with LMS filtering. Figure 5 (a) shows the raw signal considered from the standard database. The PLI noise synthetic signal is generated, the noise signal is added with the input raw ECG signal as shown in figure $5(\mathrm{~b})$. The denoising procedure is applied to
ECG MIT-BIH arrhythmia database is considered for simulation. This database contains 42 ECG recordings taken in majority by ML-II lead, sampled at $360 \mathrm{~Hz}$ with a 30 minutes duration and 11-bit resolution. Each ECG record is annotated by two experts which determined the QRS complex positions and some important indications about the normality or abnormality of these waves. The performance of the proposed system is calculated using different parameters such as SNR, MSE, CCR, and PRD. The considered dataset includes of 42 ECG signals, the system is tested for more than 20 signals and the average value is tabulated in the respective tables and also plotted in the graph.

Case. 1 : PLI Noise

In the first case, PLI noise is added to the raw ECG signal taken from the MIT-BIH arrhythmia database. To estimate the performance of the system, the raw ECG is added with PLI synthetic noise.

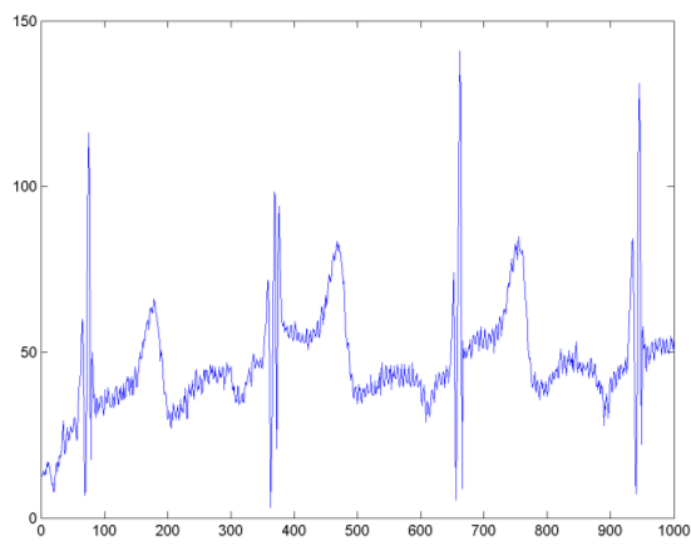

(b) Noise Signal

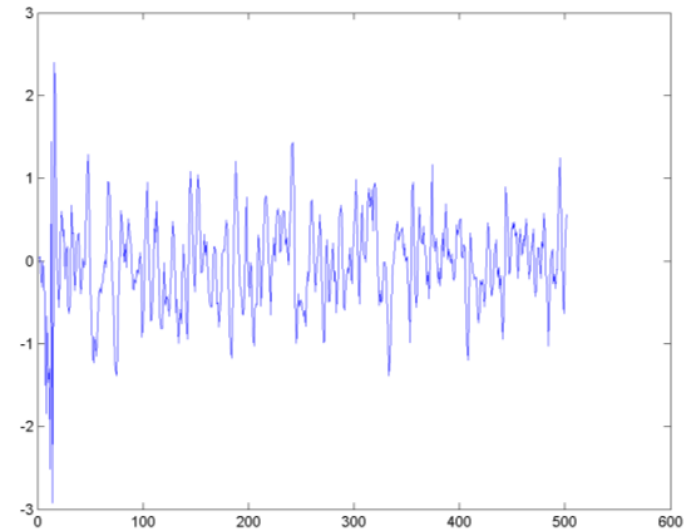

(d) LMS output signal

the noisy signal, here NLMS adaptive filtering procedure is applied to take out the PLI noise, the resulting signal is applied to LMS to get more accurate denoised signal shown in figure 5(c) and (d). Figure 5(e) and (f) shows Kalman smoothening and denoised ECG signal. 


\section{ECG Signal De-noising based on Adaptive Filters}

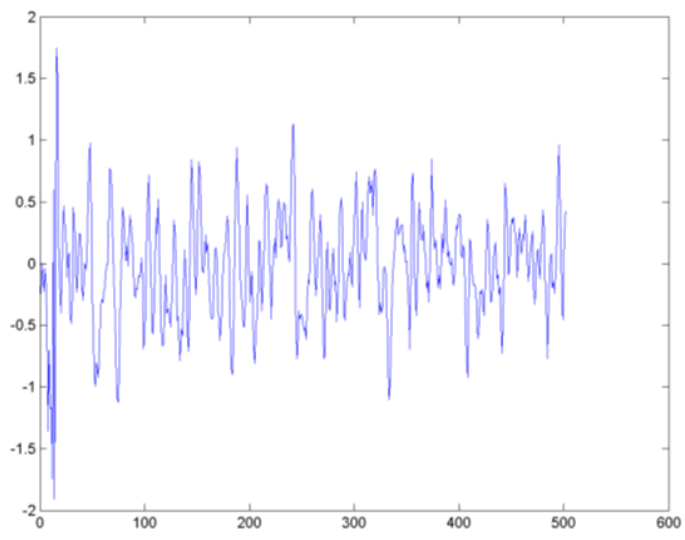

(e) Kalman smoothening

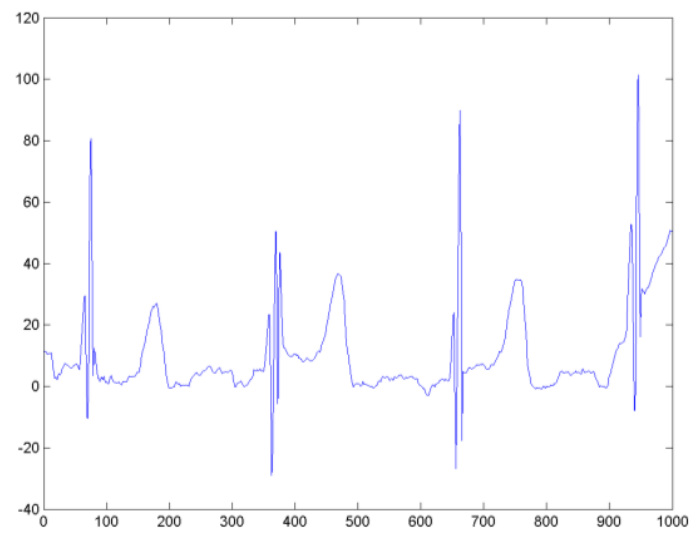

(f) Denoised signal

Figure 5: ECG Signal with PLI Noise

Case.2 BW Noise

Generally, BW noise occurs due to body movement, the improper placing of electrodes, sweat, respiration

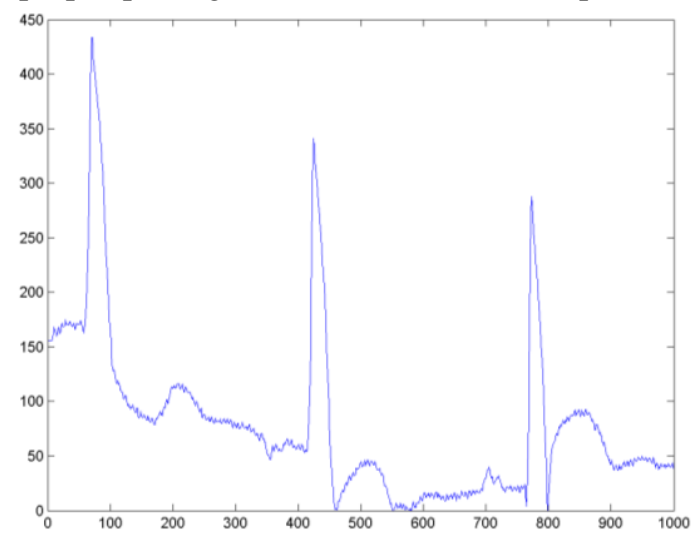

(a) Input Signal
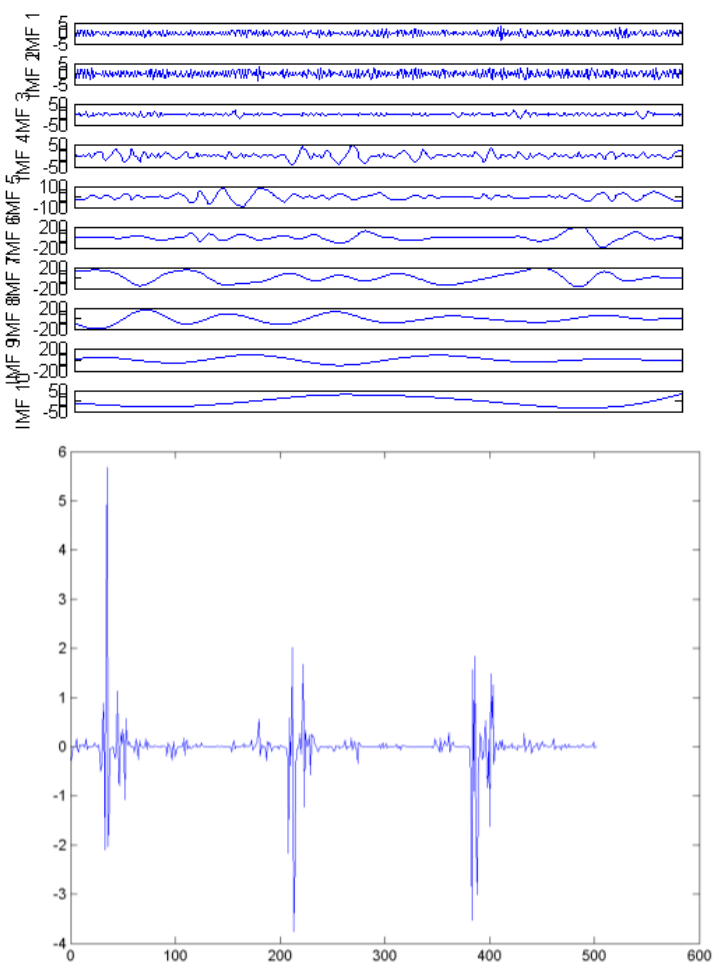

(e) Kalman Smoothening

Figure 6: ECG Signal with BW Noise or movement of the patient. Figure 6 (a) shows the raw ECG signal (person 108m) and (b) shows the BW noise added to the ECG signal.

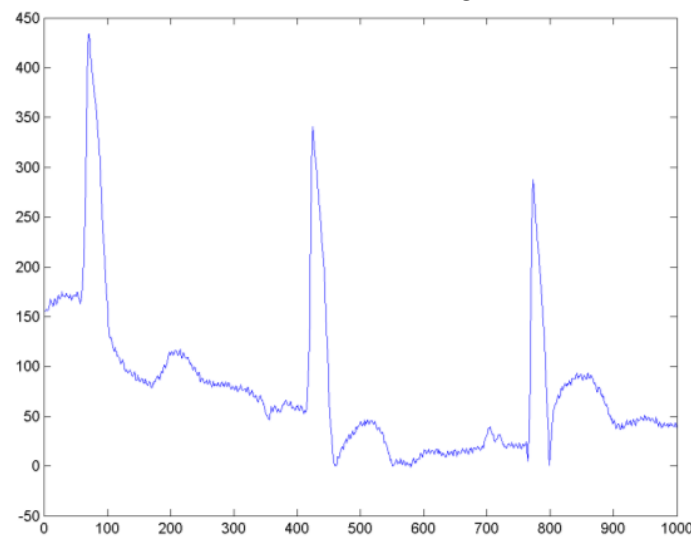

(b) Noise Signal

Figure 6: (e) CEEMD IMFs Signal

The BW noise can be eliminated using CEEMD and NLMS adaptive filtering. Figure (c) and (d) shows the resulting signal of CEEMD and NLMS filtering. Kalman smoothening signal is as shown in figure (e). The reconstructed denoised ECG signal by IDWT is as shown in figure (f)

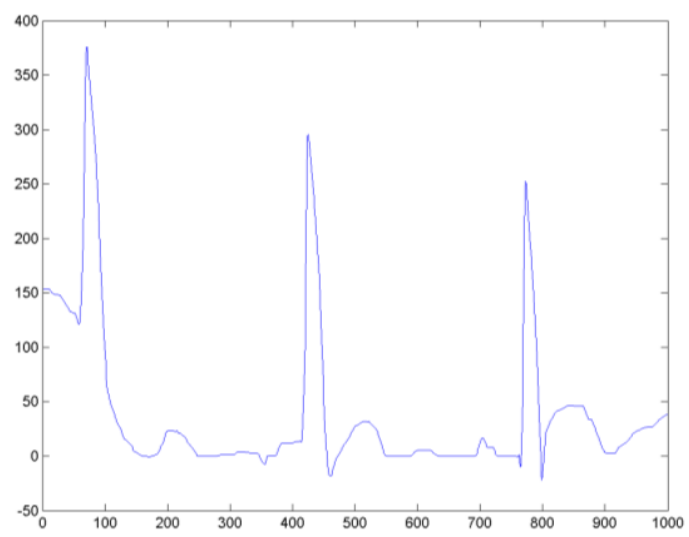

(f) Denoised 


\section{Case.3 CN Signal}

In the above cases, we have analyzed the denoising of PLI and BW noise. In this case, we are adding two different

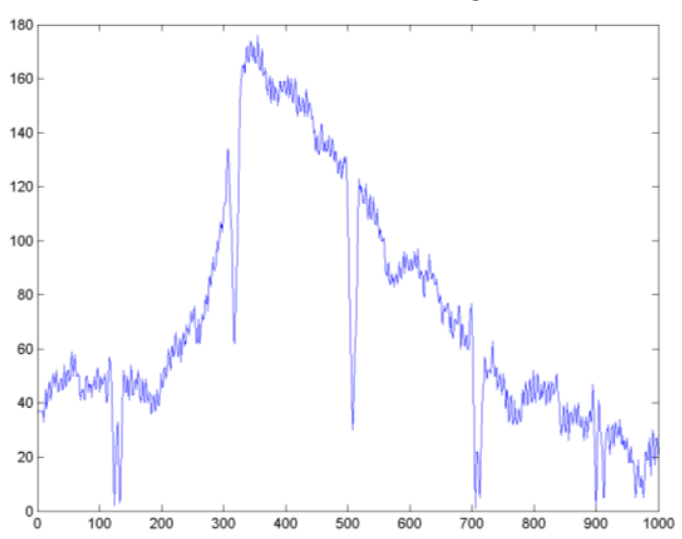

(a) Input Signal

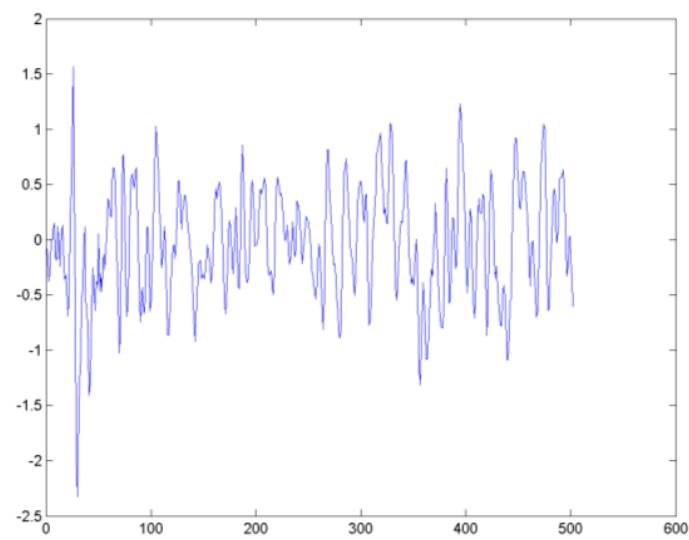

(c) NLMS output signal

Form case one and two its conclusion that the PLI noise can be denoised more accurately using NLMS and LMS adaptive filtering techniques and the BW noise can be denoised using CEEMD and NLMS filleting, hence the CN

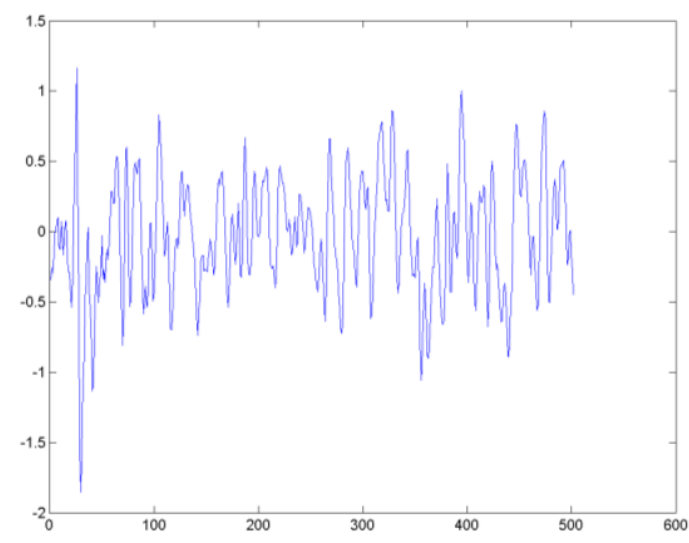

(e) Kalman Smoothening noises (PLI and BW) to generate the CN signal. Figure 7 (a) shows the input raw ECG signal (person 215m) and (b) shows the noisy signal.

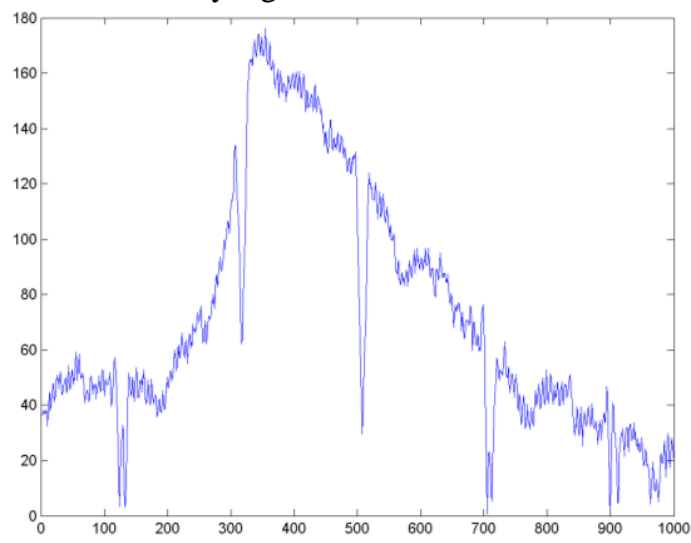

(b) Noise Signal

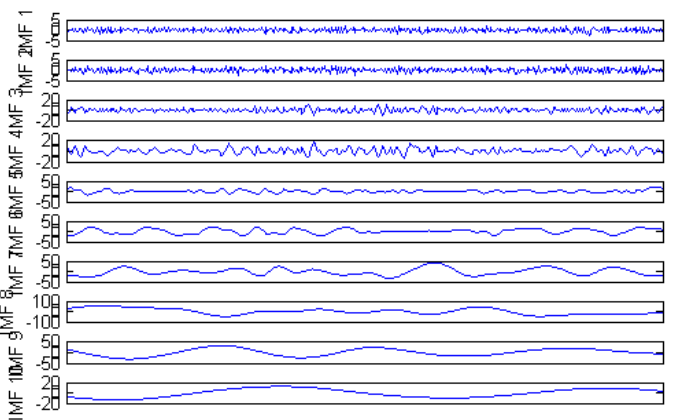

(d) CEEMD output signal

signal is denoised by the combination of all the threetechnique. NLMS, LMS, and CEEMD techniques are applied to denoise the $\mathrm{CN}$ in ECG signal as shown in the figure (c) (d) (e) and (f).

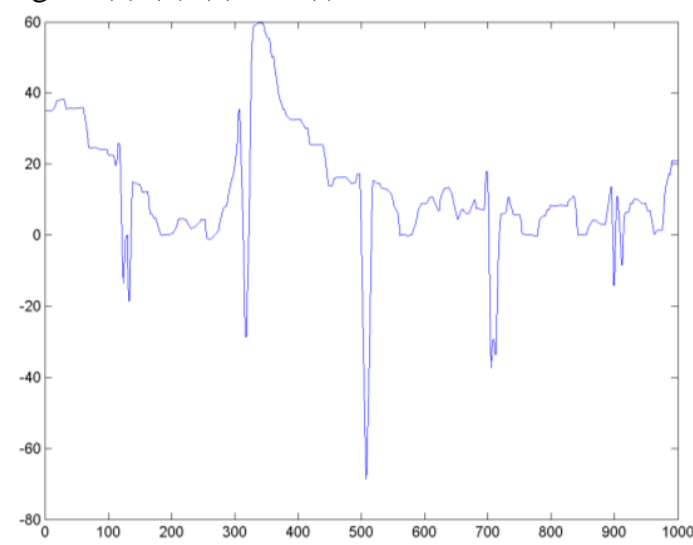

(f) Denoised

Figure 7: ECG Signal with CN Noise

SNR: SNR is used to compute the level of noise in the ECG, thus SNR value is increased, better is the amount of noise control, which is measured in $\mathrm{dB}$. The SNR is calculated using Eq. (18), Table 1, 2 and 3 shows the SNR performance calculation of PLI, BW, and CN. Figure 8 shows the graphical illustration of SNR and Figure 9 and 10 shows the graph plotted to compare the performance of the proposed system with a recently developed system. Table 4 and 5 show the SNR comparison between proposed and existing system considering BW and PLI.

Published By:

Blue Eyes Intelligence Engineering 
ECG Signal De-noising based on Adaptive Filters

$$
S N R=10 * 10 \log _{10}\left[\left|\frac{E_{E C G \text { Signal }}}{E_{\text {Noise Signal }}}\right|^{2}\right]
$$

Table 1: SNR performance for PLI

\begin{tabular}{|c|c|c|c|}
\hline S.No & Dataset Signal & SNR Before (db) & SNR After (db) \\
\hline 1 & $101 \mathrm{~m} . \mathrm{mat}$ & 21.577 & 24.68752 \\
\hline 2 & $108 \mathrm{~m} . \mathrm{mat}$ & 40.72482 & 43.29794 \\
\hline 3 & $116 \mathrm{~m} . \mathrm{mat}$ & 35.18047 & 38.81559 \\
\hline
\end{tabular}

Table 2: SNR Performance for BW

\begin{tabular}{|l|l|l|l|}
\hline 1 & $101 \mathrm{~m} . \mathrm{mat}$ & 21.32528 & 38.18381 \\
\hline 2 & $108 \mathrm{~m} . \mathrm{mat}$ & 40.46893 & 51.52472 \\
\hline 3 & $116 \mathrm{~m} . \mathrm{mat}$ & 34.93251 & 45.90352 \\
\hline
\end{tabular}

Table 3: SNR Performance for $\mathrm{CN}$ \begin{tabular}{|l|c|c|c|} 
S.No & Dataset Signal & SNR Before (db) & SNR After (db) \\
\hline
\end{tabular}

\begin{tabular}{|c|c|c|c|}
\hline 1 & $101 \mathrm{~m} . \mathrm{mat}$ & 21.47848 & 36.8563 \\
\hline 2 & $108 \mathrm{~m} . \mathrm{mat}$ & 40.60254 & 58.64563 \\
\hline 3 & $116 \mathrm{~m} . \mathrm{mat}$ & 35.08255 & 51.16709 \\
\hline
\end{tabular}

\section{\begin{tabular}{l|l|l|l} 
S.No & Dataset Signal & SNR Before (db) & SNR After (db)
\end{tabular}}

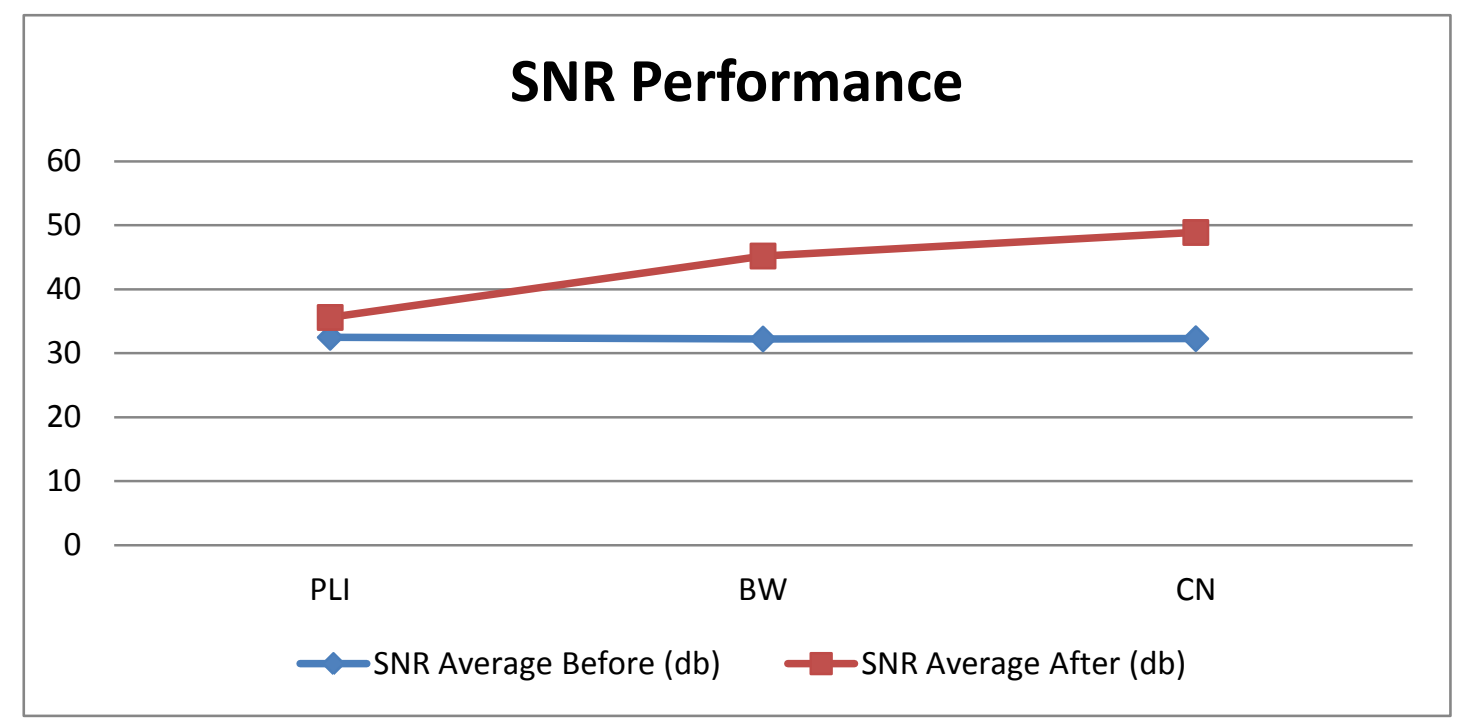

Figure 8: Overall SNR Performance of Proposed System

Table 4: Performance comparison of BW denoising

\begin{tabular}{|c|c|c|c|c|c|}
\hline S.No & Database & $\begin{array}{c}\text { Type of } \\
\text { Noise }\end{array}$ & Methods & $\begin{array}{c}\text { SNR } \\
\text { (dB) }\end{array}$ \\
\hline 1 & MIT-BIH & BW & EMD-RL & $\begin{array}{c}\text { Riemann Liouvelle Fractional Integral based Empirical Mode Decomposition } \\
\text { for ECG Denoising [23] }\end{array}$ & $\begin{array}{c}7.6487 \\
\text { Signals [16] }\end{array}$ \\
\hline 2 & MIT-BIH & $B W$ & $\begin{array}{c}\text { Non-flat Structuring } \\
\text { Element }\end{array}$ & $\begin{array}{c}\text { An Improved Algorithm for Noise Suppression and Baseline Correction of ECG } \\
\text { Morphological } \\
\text { filtering }\end{array}$ & $\begin{array}{c}\text { An Integration of Improved Median and Morphological Filtering Techniques for } \\
\text { Electrocardiogram Signal Processing [21] }\end{array}$ \\
\hline 3 & MIT-BIH & BW & NLWT & $\begin{array}{c}\text { Electrocardiogram signal denoising using non-local wavelet transform domain } \\
\text { filtering [24] }\end{array}$ \\
\hline 4 & MIT-BIH & $B W$ & CEEMD, NLMS & Proposed System \\
\hline 5
\end{tabular}

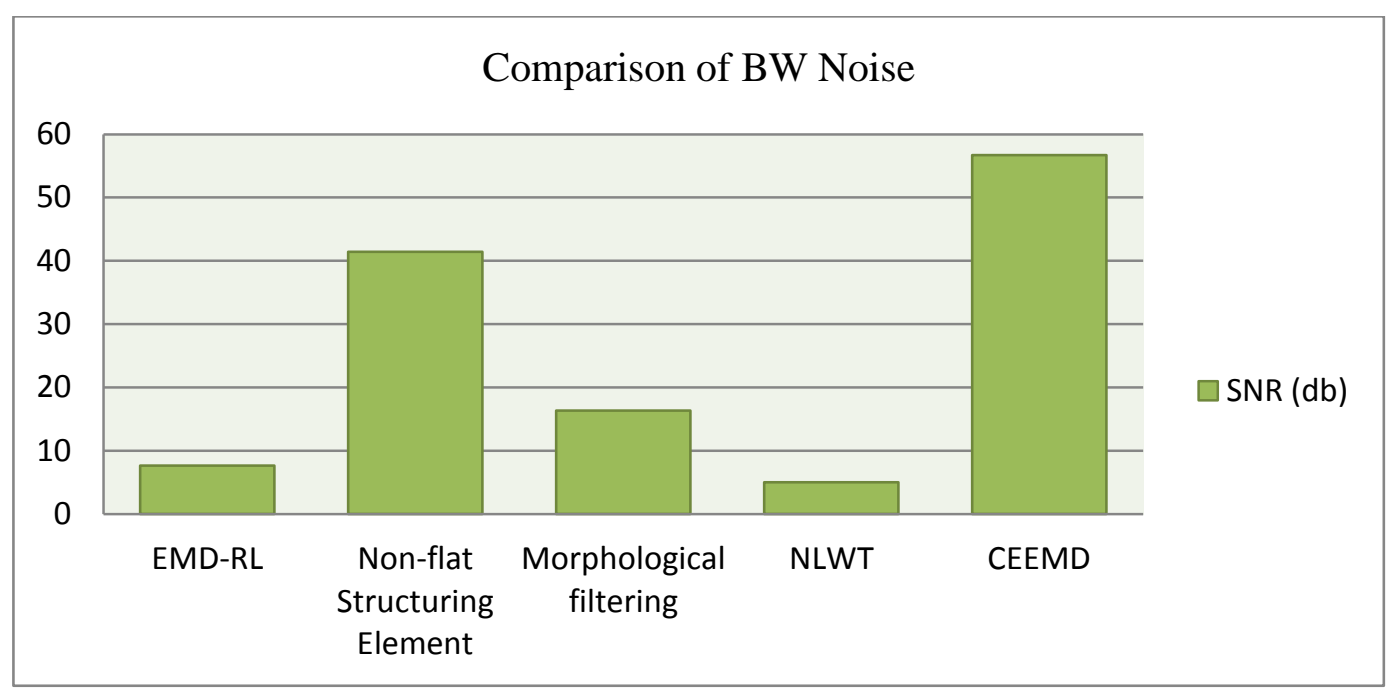

Figure 9: Comparison of BW Noise SNR 
Table 5: Performance comparison of PLI denoising

\begin{tabular}{|c|c|c|c|c|c|}
\hline S.No & Database & $\begin{array}{c}\text { Type of } \\
\text { Noise }\end{array}$ & Methods & $\begin{array}{c}\text { SNR } \\
\text { (dB) }\end{array}$ \\
\hline 1 & MIT-BIH & PLI & EMD-RL & Riemann Liouvelle Fractional Integral based Empirical Mode Decomposition for ECG \\
Denoising [23] & 12.0526 \\
\hline 2 & MIT-BIH & PLI & FIR & A New Method for Denoising ECG Signal using Sharp Cut-off FIR Filter [20] & 9.1828 \\
\hline 3 & $M I T-B I H$ & $P L I$ & NLWT & $\begin{array}{c}\text { Electrocardiogram signal denoising using non-local wavelet transform domain filtering } \\
\text { [24] }\end{array}$ \\
\hline 4 & $M I T-B I H$ & $P L I$ & NLMS,LMS & Proposed System \\
\hline
\end{tabular}

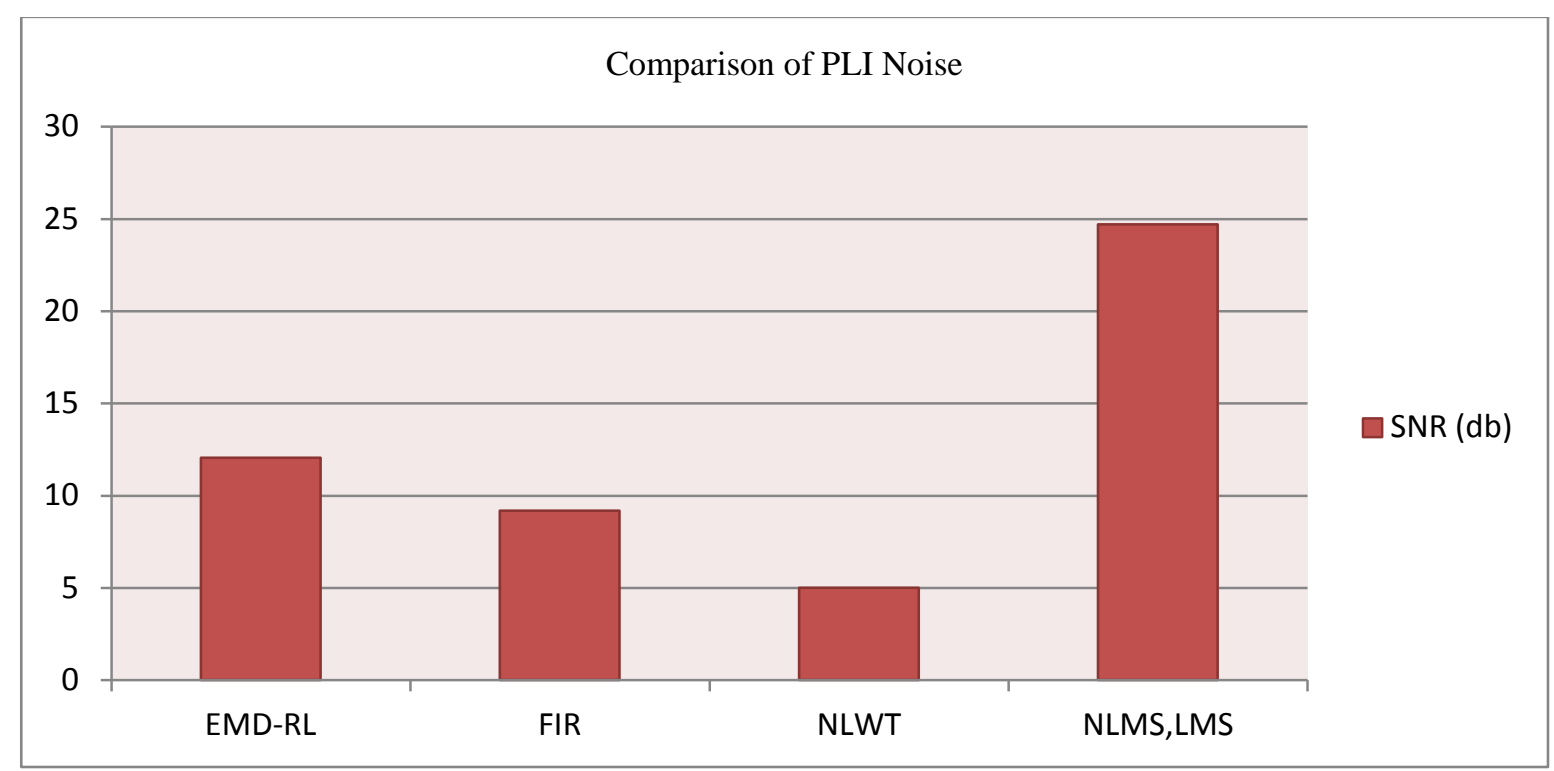

Figure 10: Comparison of PLI Noise SNR

CCR: The CCR is a statistical concept. It can measure the similarity between denoised and input signal. The $\mathrm{CC}$ is used to evaluate the methods, as is given in Eq. (19). Table 6 shows the performance of the proposed system and Figure 11 shows the graphical representation of CCR for different noises.

$$
C C R=\sum_{n=0}^{N-1} \frac{x(n) x(n)}{\sqrt{\sum_{n=0}^{N} x^{2}(n) \sum_{n=0}^{N} x^{2}(n)}}
$$

Table 6: CCR Performance

\begin{tabular}{|c|c|c|c|c|}
\hline \multirow{2}{*}{ S.No } & \multirow{2}{*}{ Dataset Signal } & \multicolumn{3}{|c|}{ CCR } \\
\cline { 3 - 5 } & & PLI & BW & CN \\
\hline 1 & $101 \mathrm{~m} . \mathrm{mat}$ & 0.997 & 0.999 & 0.999 \\
\hline 2 & $108 \mathrm{~m} . \mathrm{mat}$ & 0.999 & 0.999 & 0.999 \\
\hline 3 & $116 \mathrm{~m} . \mathrm{mat}$ & 0.999 & 0.999 & 0.999 \\
\hline 4 & Average & 0.997 & 0.999 & 0.985 \\
\hline
\end{tabular}

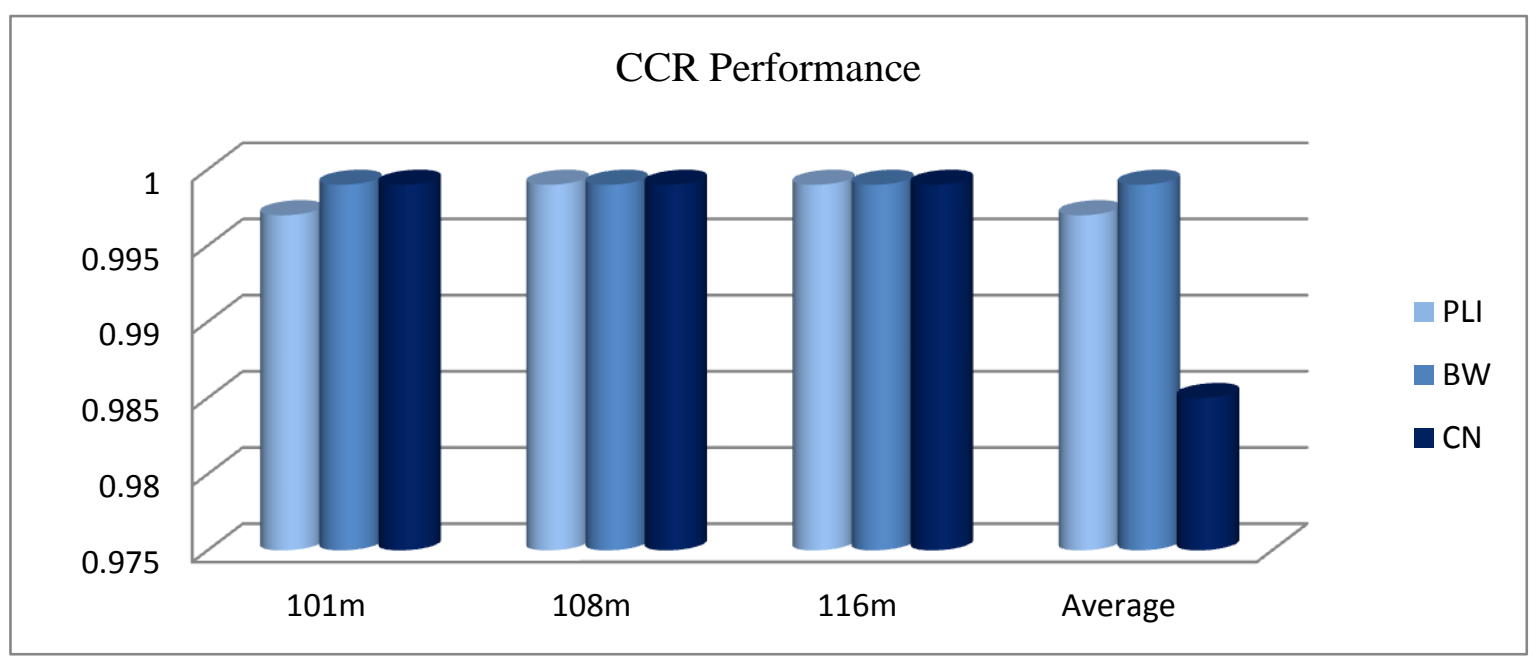

Figure 11: CCR Performance Graph

MSE: MSE denotes the deviation of the reconstructed signal from the input. Hence lower the value of MSE better is the quality of the reconstructed signal. The Eq. (20) represents the MSE calculation. Table 7 shows the performance of the proposed system and Figure 12 shows a graphical demonstration of MSE for different noises.

$$
M S E=\sqrt{\frac{1}{N}} * \sum_{n=0}^{N-1}[x(n)-x(n)]^{2}
$$




\section{ECG Signal De-noising based on Adaptive Filters}

Table 7: MSE Performance

\begin{tabular}{|c|c|c|c|c|}
\hline \multicolumn{4}{|c|}{ Table 7: MSE Performance } \\
\cline { 3 - 5 } $\mid$ S.No & Dataset Signal & \multicolumn{3}{|c|}{ MSE } \\
\cline { 3 - 5 } & & PLI & BW & CN \\
\hline 1 & $101 \mathrm{~m} . \mathrm{mat}$ & 0.119 & 0.005 & 0.007 \\
\hline
\end{tabular}

\begin{tabular}{|c|c|c|c|c|}
\hline 2 & $108 \mathrm{~m} . \mathrm{mat}$ & 0.135 & 0.020 & 0.003 \\
\hline 3 & $116 \mathrm{~m} . \mathrm{mat}$ & 0.105 & 0.020 & 0.006 \\
\hline 4 & Average & 0.286 & 0.118 & 0.213 \\
\hline
\end{tabular}

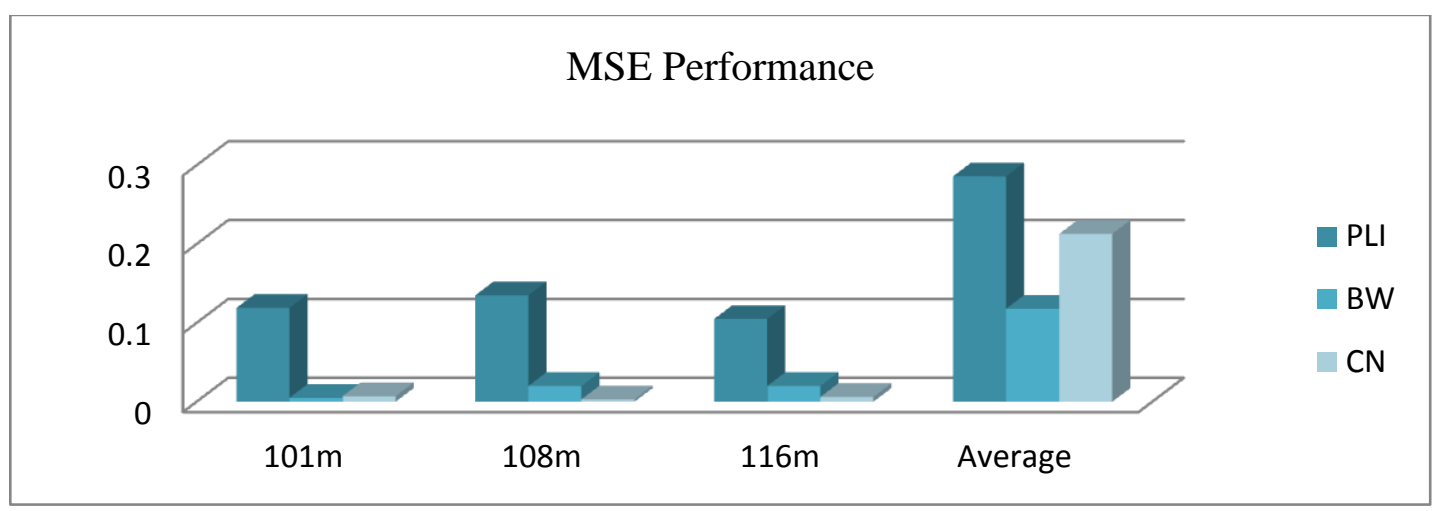

Figure 12: MSE Performance Graph

PRD: PRD is considered as a quality measure and is used to evaluate the reliability of the reconstructed signal. Table 8 shows the performance of the proposed system and Figure 13 shows a graphical depiction of PRD for different noises.

$$
\left.P R D=100 * \sqrt{\left\{\frac{\sum_{n=1}^{N}[x(n)-\hat{X}(n)]^{2}}{\sum_{n=1}^{N}[x(n)]^{2}}\right.}\right\}
$$

Table 8: PRD Performance

\begin{tabular}{|c|c|c|c|c|}
\hline \multirow{2}{*}{ S.No } & \multirow{2}{*}{ Dataset Signal } & \multicolumn{3}{|c|}{ PRD } \\
\cline { 3 - 5 } & & PLI & BW & CN \\
\hline 1 & $101 \mathrm{~m} . \mathrm{mat}$ & 5.801 & 1.231 & 1.434 \\
\hline 2 & $108 \mathrm{~m} . \mathrm{mat}$ & 0.684 & 0.265 & 0.116 \\
\hline 3 & $116 \mathrm{~m} . \mathrm{mat}$ & 1.1453 & 0.506 & 0.276 \\
\hline 4 & Average & 3.066 & 0.859 & 4.258 \\
\hline
\end{tabular}

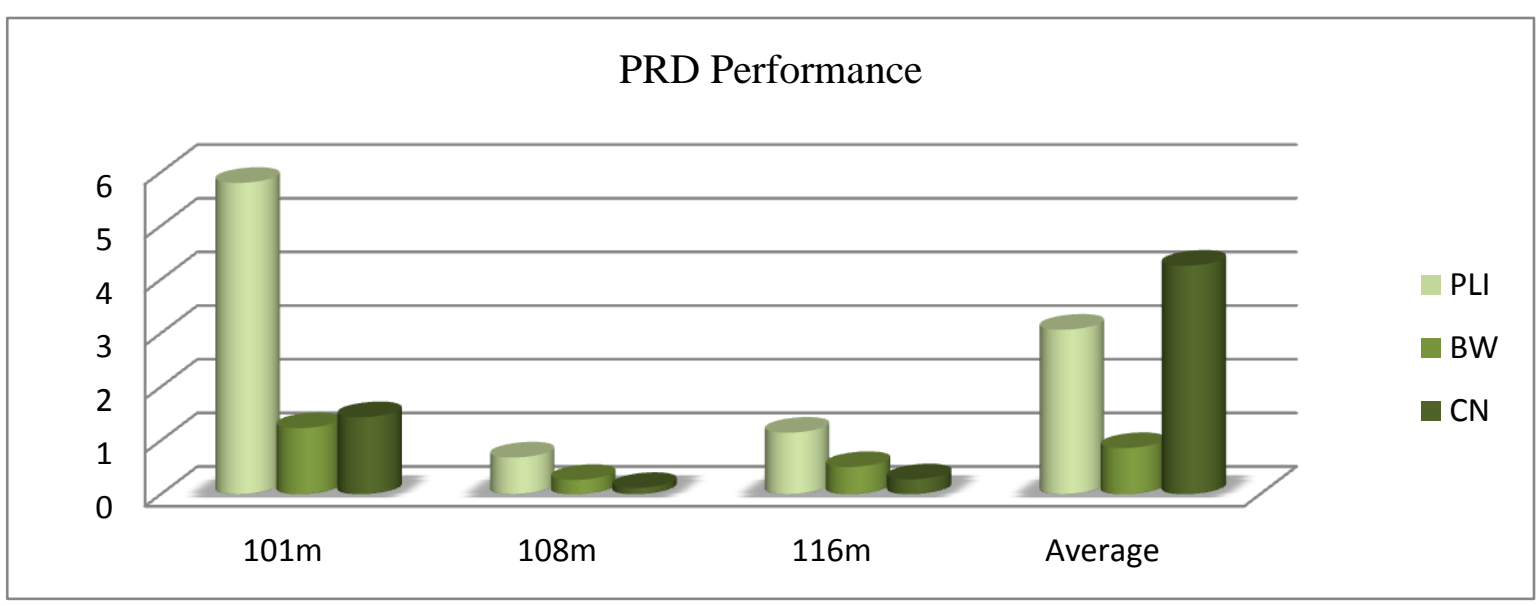

Figure 13: PRD Performance Graph

\section{CONCLUSION}

The ECG plays a crucial role in diagnosis and reflecting all cardiac diseases however, at the moment of obtaining it, different kind of noise interferes with the signal. In the proposed paper denoising of ECG waveform for different noise is proposed. ECG is denoised using CEEMD, LMS, NLMS method. The DWT consists of decomposing any non-stationary data in different frequency sub-bands. The PLI noise is removed using the NLMS and LMS adaptive filtering, BW noise signal is denoised using CEEMD and NLMS technique. The $\mathrm{CN}$ is eliminated by CEEMD, NLMS, and LMS filtering techniques. Kalman smoother is applied for smooth output, reconstructing the denoised signal using IDWT. The performance of the system is evaluated using SNR, MSE, PDR and CCR parameters. Comparing the performance of the proposed system with the existing system, the proposed system gives better performance than the existing system. The signal quality is improved by the proposed system.

\section{REFERENCE}

1. Abdalla Y, Zhao Y, and Wu, L., "Denoising ECG Signal by Complete EEMD Adaptive Noise", In 2017 IEEE International Symposium on Signal Processing and Information Technology (ISSPIT). IEEE, pp. 337-342, 2017

2. Ning $X$ and Selesnick I W, "ECG Enhancement and QRS Detection Based on Sparse Derivatives", Elsevier, Vol. 8, No. 6, pp.713-23, 2013.

3. Biswas, U., Das, A., Debnath, S. and Oishee, I., "ECG Signal Denoising by using Least-Mean-Square and Normalised-Least-MeanSquare Algorithm based Adaptive Filter", In 2014 Internationa Conference on Informatics, Electronics \& Vision (ICIEV). IEEE, pp. $1-6,2014$

4. Wu S, Shen Y, Zhou Z, Lin L, Zeng Y, and Gao X, "Research Of Fetal ECG Extraction Using Wavelet Analysis And Adaptive Filtering", Elsevier, Vol.43, No. 10, pp. 1622-7, 2013.

5. Sharma, S. and Narwaria, R.P., "Noise Reduction from ECG Signal using Adaptive Filter Algorithm", International 
Journal of Engineering Research \& Technology (IJERT), Vol. 3, Issue 7, 2014

6. Mishu M M, Hossain A A, and Emon M E, "De-Noising Of ECG Signals Using Dual-Tree Complex Wavelet Transform", IEEE, pp. 379-382, 2014.

7. Joshi S L, Vatti R A, and Tornekar R V, "A Survey on ECG Signal De-Noising Techniques", IEEE, pp. 60-64, 2013.

8. Sadhukhan D, Mitra M, "ECG Noise Reduction Using Linear and Non-Linear Wavelet Filtering", Research gate, pp. 22-23, 2014.

9. Jenitta, J. and Rajeswari, A., "Denoising of ECG Signal based on Improved Adaptive Filter with EMD and EEMD", In Information \& Communication Technologies (ICT), 2013 IEEE Conference. IEEE, pp. 957-962, 2013

10. Belgurzi, A.N.S., and Elshafiey, I., "A Power Line Interference Canceler using Wavelet Transform and Adaptive Filter for ECG Signal", In Computer and Applications (ICCA), 2017 International Conference. IEEE, pp. 206-210, 2017

11. Guleria R and Kaur R, "Removing the Power Line Interference From ECG Signal Using Kalman Least Mean Square Filter", IEEE, pp. 1151-1157, 2016.

12. Shemi, P.M. and Shareena, E.M., "Analysis of ECG Signal Denoising using Discrete Wavelet Transform", In Engineering and Technology (ICETECH), 2016 IEEE International Conference. IEEE, pp. 713718,2016

13. Zhou, Y., Hu, X., Tang, Z. and Ahn, A.C., "Denoising and Baseline Correction of ECG Signals using Sparse Representation", In Signal Processing Systems (SiPS). IEEE, pp. 1-6, 2016

14. Gilani, S.O., Ilyas, Y. and Jamil, M., "Power Line Noise Removal from ECG Signal using Notch, Band Stop and Adaptive Filters", In Electronics, Information, and Communication (ICEIC), 2018 International Conference. IEEE, pp. 1-4, 2018

15. Biswas, U., and Maniruzzaman, M., "Removing Power Line Interference from ECG Signal using Adaptive Filter and Notch Filter", In Electrical Engineering and Information \& Communication Technology (ICEEICT), 2014 International Conference. IEEE, pp. 14, 2014

16. Verma, R., Mehrotra, R., and Bhateja, V., "An Improved Algorithm for Noise Suppression and Baseline Correction of ECG Signals", In Proceedings of the International Conference on Frontiers of Intelligent Computing: Theory and Applications (FICTA). Springer, pp. 733-739, 2013

17. Sharma, I., Mehra, R. and Singh, M., "Adaptive Filter Design for ECG Noise Reduction using LMS Algorithm", In Reliability, Infocom Technologies and Optimization (ICRITO)(Trends and Future Directions), 2015 4th International Conference. IEEE, pp. 1-6, 2015

18. Sharma, B. and Suji, R.J., "ECG Denoising using Weiner Filter and Adaptive Least Mean Square Algorithm", In Recent Trends in Electronics, Information \& Communication Technology (RTEICT), IEEE International Conference. IEEE, pp. 53-57, 2016

19. Yadav, S.K., Sinha, R. and Bora, P.K., "Electrocardiogram Signal Denoising using Non-Local Wavelet Transform Domain Filtering”, IET Signal Processing. IEEE, Vol. 9, Issue 1, pp.88-96, 2015

20. Roy, S. and Chandra, A., "A New Method for Denoising ECG Signal using Sharp Cut-off FIR Filter", In 2018 International Symposium on Devices, Circuits, and Systems (ISDCS). IEEE, pp. 1-6, 2018

21. Verma, R., Mehrotra, R., and Bhateja, V., "An Integration of Improved Median and Morphological Filtering Techniques for Electrocardiogram Signal Processing", In Advance Computing Conference (IACC). IEEE, pp. 1223-1228, 2013

22. Patro, K.K. and Kumar, P.R., "De-noising of ECG Raw Signal by Cascaded Window-based Digital Filters Configuration", In Power, Communication and Information Technology Conference (PCITC). IEEE, pp. 120-124, 2015

23. Jain, S., Bajaj, V. and Kumar, A., "Riemann Liouvelle Fractional Integral based Empirical Mode Decomposition for ECG Denoising", IEEE, Vol. 22, Issue 4, 2017

24. Yadav S.K, Sinha R. and Bora, P.K, "Electrocardiogram signal denoising using non-local wavelet transform domain filtering", IET Signal Processing, Vol. 9, Issue 1, pp.88-96, 2015

\section{AUTHORS PROFILE}

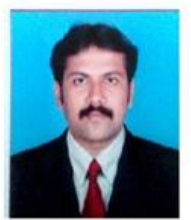

Keshavamurthy $\mathrm{T} G$ received his $\mathrm{BE}$ degree in Telecommunication Engineering from AMC Engineering College, Bangalore and M.Tech in Digital Electronics and Communication Systems from Malnad College of Engineering, Hassan. Presently, he is pursuing his $\mathrm{PhD}$ from SSAHE, Tumkur and Working as an Assistant professor in
Electronics and Communication Department, Sri Siddhartha Institute of Technology, Tumkur, India.

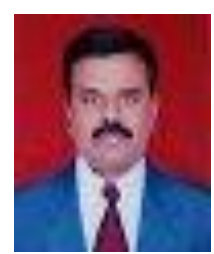

M.N Eshwarappa received his BE degree in Electrical and Electronics Engineering from MCE, Hassan and M.Tech in Industrial Electronics KREC-Surthkal, Manglore University. M.N Eshwarappa was awarded the $\mathrm{PhD}$ degree in 2013 for his work in the area of signal processing. Presently, he is Working as a professor in Electronics and Communication Department, Sri Siddhartha Institute of Technology, Tumkur, India 